Artículo de posesión

\title{
La respuesta inmunitaria adaptativa en la infección crónica por Trypanosoma cruzi
}

\author{
John M. González ${ }^{1, *}$, Adriana Cuéllar², Concepción J. Puerta ${ }^{3}$ \\ ${ }^{1}$ Grupo de Ciencias Básicas Médicas, Facultad de Medicina, Universidad de los Andes, Bogotá, Colombia \\ ${ }^{2}$ Grupo de Inmunobiología y Biología Celular, Departamento de Microbiología, Facultad de Ciencias, \\ Pontificia Universidad Javeriana, Bogotá, Colombia \\ ${ }^{3}$ Laboratorio de Parasitología Molecular, Departamento de Microbiología, Facultad de Ciencias, \\ Pontificia Universidad Javeriana, Bogotá, Colombia
}

Artículo de posesión para el ingreso como miembro correspondiente de la

Academia Colombiana de Ciencias Exactas, Físicas y Naturales el 27 de septiembre de 2017

\begin{abstract}
Resumen
La infección por Trypanosoma cruzi causa la enfermedad de Chagas, la cual presenta una fase aguda en la que la activación del sistema inmunitario ayuda a controlar el parásito. No obstante, el parásito puede persistir en bajos niveles y producir una fase crónica en la cual el 70 \% de los individuos infectados permanece asintomático, en tanto que los demás presentan compromiso tisular, principalmente en el corazón, y pueden desarrollar una miocardiopatía inflamatoria crónica. Durante el proceso de infección la respuesta inmunitaria tiene un papel importante en la protección, pero también se ha asociado con la patogenia, especialmente en la fase crónica. Los estudios del grupo de investigación sobre la enfermedad de Chagas conformado por investigadores de la Universidad de los Andes y la Pontificia Universidad Javeriana en Colombia, se han centrado en analizar la respuesta inmunitaria adaptativa inducida frente a T. cruzi. En este artículo, se resumen y analizan los resultados de la evaluación de la respuesta inmunitaria humoral contra la proteína KMP-11 del parásito, así como de la respuesta inmunitaria celular en sangre periférica de pacientes con cardiopatía chagásica crónica. La funcionalidad de los anticuerpos específicos y el porcentaje de linfocitos $\mathrm{T}$ específicos de antígeno en sangre periférica y sus marcadores de funcionalidad se evaluaron usando un péptido de nueve aminoácidos de la proteína KMP-11 denominado TcTLE. (C) 2017. Acad. Colomb. Cienc. Ex. Fis. Nat.
\end{abstract}

Palabras claves: Enfermedad de Chagas; Trypanosoma cruzi; Linfocitos T; Anticuerpos.

Adaptive immune response in chronic infection by Trypanosoma cruzi

\begin{abstract}
Trypanosoma cruzi infection causes Chagas disease, which presents an acute phase where the activation of the immune system can help to control the parasite. However, the parasite persists at a low level leading to a chronic phase where $70 \%$ of the infected individuals remain asymptomatic, and the others have tissue involvement, mainly a chronic inflammatory cardiomyopathy. During the infection process, the immune response plays an important role in protection, but it has also been associated with pathogenesis, especially during the chronic phase. The studies carried out by the Chagas disease research group from Universidad de los Andes and the Pontificia Universidad Javeriana in Colombia, have been focused on dissecting the adaptive immune response induced against $T$. cruzi. Here we summarize and analyze the results of the evaluation of the humoral immune response against the KMP-11 protein from the parasite. We also describe the cellular immune response against $T$. cruzi in peripheral blood of patients with chronic Chagas heart disease. We evaluated the possible role of peptide-specific antibodies and the percentage of peripheral blood antigen-specific lymphocytes and their surface markers using a nine amino acids peptide from the KMP-11 protein termed TcTLE. (C) 2017. Acad. Colomb. Cienc. Ex. Fis. Nat.
\end{abstract}

Key words: Chagas disease; Trypanosoma cruzi; T lymphocytes; Antibodies.

\section{Generalidades de la enfermedad de Chagas}

La enfermedad de Chagas es causada por la infección con el parásito flagelado Trypanosoma cruzi. Esta es una de las enfermedades desatendidas que más retos plantea debido a su gran impacto socioeconómico (Castillo, et al., 2008), y a su compleja historia natural en la que aparecen diversas formas de transmisión, cuadros clínicos, y genotipos del parásito (unidades discretas de tipificación, o Discrete Typing Units, DTU), así como la participación de diferentes

*Correspondencia:

John M. González, johgonza@uniandes.edu.co

Recibido: 09 de julio de 2017

Aceptado: 19 de diciembre de 2017 
mecanismos de daño tisular durante la fase crónica. La infección puede ocurrir por trasmisión vectorial o por consumo de líquidos que contengan la forma infectiva del parásito, el tripomastigote metacíclico, pero también por infección congénita, trasplante, reactivación en personas inmunosuprimidas, transfusión sanguínea o accidente de laboratorio (Bern, 2015). Durante el periodo agudo de la enfermedad, que puede durar entre seis y ocho semanas, la mayoría de los casos son asintomáticos, en tanto que los individuos sintomáticos presentan síntomas constitutivos como fiebre, malestar general y cefalea. En ocasiones, se pueden presentar signos característicos de la enfermedad, como el signo de Romaña (edema palpebral unilateral) y el chagoma (nódulo inflamatorio en la piel), causados por la inflamación y que indican la vía de entrada del parásito. En la fase aguda se pueden detectar parásitos en sangre periférica. Aunque el parásito puede ser controlado por el sistema inmunitario, suele persistir en bajos niveles en las personas infectadas crónicamente. Así, los individuos entran en una fase crónica asintomática (indeterminada) en la que el diagnóstico se hace principalmente mediante pruebas serológicas que determinan la presencia de anticuerpos anti-T. cruzi en sangre periférica (Bern, 2015; Rosas, et al., 2012). Las personas inmunosuprimidas (por ejemplo, con HIV/SIDA) que presentan la infección por $T$. cruzi, pueden presentar reactivación de la infección, lo que demuestra el papel de los linfocitos T CD4+ en el control del parásito (de Freitas, et al., 2011). La fase asintomática puede extenderse por años o décadas, sin embargo, cerca del $30 \%$ de los individuos puede entrar en la fase crónica sintomática (determinada) en la cual el parásito presenta tropismo hacia ciertos tejidos (Sabino, et al., 2013). En individuos con infección crónica sintomática, se estima que el compromiso cardiaco ocurre en cerca del 90 \%, casos en que el parásito se replica en los cardiomiocitos, y el digestivo (megacolón o megaesófago) en el 10 \%, casos en los que el parásito se replica en las células de sistema nervioso periférico (Bern, 2015).

La patogenia de la enfermedad crónica no se conoce de forma precisa, pero se ha determinado que la persistencia del parásito, su genotipo o DTU, y la disfunción del sistema inmunitario pueden ser parte del proceso de daño tisular (Bern, 2015; Klahr, et al., 2016; Rosas, et al., 2012). Si bien existe un tratamiento antiparasitario con dos medicamentos, el benzinidazol y el nifurtimox, ambos se recomiendan para pacientes en fase aguda de la infección, incluidas las mujeres en gestación, las personas con infección oral y cuando hay reactivación, pero no en aquellas con infección crónica (Bern, et al., 2007). En un estudio clínico, el tratamiento con benzinidazol en pacientes con cardiopatía establecida demostró que, a pesar de la disminución o control de la parasitemia, no había mejoría clínica en los pacientes (Morillo, et al., 2015). Estos resultados evidencian la necesidad de encontrar alternativas de tratamiento y de estudiar nuevos mecanismos inductores de la enfermedad, como los inmunitarios, los cuales operarían durante la infección crónica con compromiso tisular (Rassi, et al., 2017).

\section{Algunos aspectos del ciclo de vida de Trypanosoma cruzi en los humanos}

Los tripomastigotes son formas del parásito que no se dividen, por lo tanto, deben invadir o circular para encontrar una célula donde adherirse y continuar el ciclo. Una vez dentro de la célula, se desarrollan los amastigotes, los cuales se multiplican por división binaria (Brener, 1973; Campo, et al., 2016). En el sitio de inoculación, los tripomastigotes usualmente invaden células fagocíticas, como los macrófagos, y células no fagocíticas, como los fibroblastos (de Souza, et al., 2010; Rosestolato, et al., 2002). Aunque se han identificado algunos ligandos que el parásito utiliza para unirse a las células huéspedes, son múltiples las moléculas que participan en esta interacción (Magdesian, et al., 2001). Después de la unión a la membrana celular, se presenta la formación de la vacuola parasitofora, donde operan diferentes mecanismos de endocitosis (Barrias, et al., 2013). Una vez dentro de la célula, empieza la formación de los amastigotes, los cuales pasan de la vacuola al citoplasma y entran en contacto directo con los organelos de las células huéspedes (Ley, et al., 1990; de Souza, et al., 2010). Ya que una de la formas del parásito es extracelular, los anticuerpos contra antígenos de superficie son uno de los mecanismos de defensa del huésped para lisar los tripomastigotes o evitar la invasión de las células (Krettli \& Brener, 1976). Asimismo, las proteínas derivadas de los amastigotes libres en el citoplasma entran en la vía de procesamiento de antígenos de clase I y pueden ser presentadas a los linfocitos T CD8+, lo cual es importante para el control de la infección (Tarleton, 2015).

La respuesta inmunitaria frente al parásito durante la infección aguda controla la parasitemia, aunque no la elimina completamente (Sosa-Estani, et al., 1998). En la fase crónica especialmente, la respuesta inmunitaria adaptativa parece tener un papel dual al controlar parcialmente la infección en individuos asintomáticos y participar en la patogenia del daño tisular en individuos sintomáticos. En este artículo se presentan los resultados obtenidos en el estudio de la respuesta inmunitaria adquirida, tanto la humoral contra la proteína KMP-11 y su péptido TcTLE, como la celular mediada por linfocitos $\mathrm{T}$ contra dicho péptido y el antígeno completo del parásito durante la infección crónica por T. cruzi. En la respuesta humoral contra un antígeno específico se ha observado que los anticuerpos pueden unirse a los tripomastigotes y disminuir la infección de células in vitro. En la respuesta celular inmunitaria se describen los cambios en los marcadores de superficie y en los funcionales de los linfocitos $\mathrm{T}$ en sangre periférica, los cuales presentaron características de agotamiento celular, asociado probablemente con la persistencia del antígeno en la enfermedad de Chagas crónica. 


\section{La respuesta inmunitaria durante la infección por Trypanosoma cruzi}

Aunque la respuesta inmunitaria se ha estudiado en las fases aguda y crónica de la enfermedad, la mayoría de estudios en humanos se han hecho en la fase crónica, especialmente porque la infección aguda por transmisión vectorial se presenta en niños de áreas endémicas y en un alto porcentaje de ellos puede cursar de forma asintomática (Rassi, et al., 2010). No obstante, en los casos de transmisión oral hay un alto porcentaje de sintomáticos (Noya, et al., 2015). Asimismo, debe considerarse la diversidad del parásito, con siete DTUs y al menos 12,000 potenciales proteínas en su genoma, lo que dificulta el estudio de la respuesta inmunitaria específica contra un antígeno (Acevedo, et al., 2017). Dado lo prolongado del tiempo en el que puede aparecer el compromiso tisular en individuos con infección y asintomáticos, el cual puede ser de años o décadas, es difícil hacer el seguimiento de un individuo durante todo el curso de la enfermedad. Por lo tanto, una de las estrategias es obtener y comparar muestras (anticuerpos o células) de individuos con infección crónica en las fases asintomática y sintomática, especialmente con compromiso cardiaco. En estos trabajos, los pacientes se clasificaron en las cuatro categorías (A-D) establecidas por la American Heart Association (AHA) (Bern, et al., 2007). Para fines prácticos, y con el objetivo de comparar los resultados, los individuos en los grupos A y B de dicha clasificación se consideraron asintomáticos (indeterminados), y los de los grupos C y D, como sintomáticos (determinados) (Giraldo, et al., 2011). La mayoría de los estudios sobre inmunidad celular en estos individuos se hacen en sangre periférica y en tejido cardiaco, y los resultados se obtienen sobre todo mediante citometría de flujo e inmunohistoquímica, respectivamente. No obstante, en algunos estudios se han analizado linfocitos derivados de corazón utilizando citometría de flujo (Giraldo, et al., 2013).

\section{La respuesta inmunitaria innata en fase crónica}

Se conoce poco del papel de los leucocitos polimorfonucleares en fase crónica, sin embargo, los eosinófilos se han asociado con el daño tisular en la respuesta crónica y se los ha detectado en el infiltrado de leucocitos en tejido cardiaco de pacientes con miocardiopatía. Se ha postulado que los productos solubles tóxicos de los eosinófilos inducen la muerte de los miocardiocitos (Molina \& Kierszenbaum, 1987). En pacientes con infección crónica por T. cruzi también se ha descrito un aumento de las metaloproteinasas 2 (MMP2) y 9 (MMP9) en neutrófilos y monocitos, comparadas con las de individuos no infectados, aunque sin diferencias significativas entre asintomáticos y sintomáticos. Estas enzimas, que participan en el remodelamiento tisular, podrían estar asociadas al daño cardiaco (Medeiros, et al., 2017)

En niños en fase asintomática temprana se ha determinado que el número de células NK y de monocitos inflamatorios (CD14+ CD16+ HLA-DR++) en sangre periférica aumenta en comparación con individuos no infectados. En estas poblaciones, cuyos valores pueden volver a niveles basales en sangre periférica en la infección crónica, se han asociado al mantenimiento de la fase asintomática (Vitelli-Avelar, et al., 2006). Sin embargo, en individuos en fase crónica sintomática se encuentran alteraciones funcionales de las poblaciones de monocitos y células dendríticas, ambas presentadoras de antígenos profesionales. El parásito puede influenciar o alterar el comportamiento de estas células a través de la infección directa (Cuellar, et al., 2009; Magalhães, et al., 2015) o mediante la activación de receptores de reconocimiento de patrones (pattern recognition receptor, PRR) y de receptores de tipo toll (toll-like receptor, TLR), o los receptores intracelulares de tipo nod (nod-like receptor, NRL) (Dey, et al., 2014; Dos-Santos, et al., 2016). La activación de estas células presentadoras de antígeno, como los monocitos y macrófagos, tiene que ver con la producción de moléculas efectoras como el óxido nítrico (NO), los productos reactivos del oxígeno (reactive oxygen species, ROS) y las citocinas inflamatorias, las cuales tienen un papel fundamental en la presentación antigénica, así como en la activación subsecuente de la respuesta mediada por linfocitos T. El parásito suele disminuir dicha respuesta alterando la actividad de la enzima NADPH oxidasa, la cual regula la producción de ROS (Dey, et al., 2014). Asimismo, los antígenos derivados del parásito alteran el patrón de citocinas producidas, por ejemplo, las células dendríticas de pacientes con enfermedad de Chagas crónica producen más interleucina 10 (IL-10) que interleucina 12 (IL-12) en presencia de la proteína de choque térmico de $T$. cruzi. La IL-12 fomenta la diferenciación de linfocitos T, en tanto que la IL-10 inhibe una presentación antigénica efectiva (Cuellar, et al., 2008). Como muchas otras enfermedades infecciosas crónicas, la calidad de la respuesta inmunitaria inducida inicialmente es fundamental para el desarrollo de una respuesta adaptativa, tanto de anticuerpos como de linfocitos $\mathrm{T}$ efectores y de memoria. Como se ha descrito, en algunos de los elementos de la respuesta inmunitaria innata en la infección por $T$. cruzi el parásito parece manipularla y alterarla a favor de su supervivencia.

\section{La respuesta inmunitaria adaptativa}

Los anticuerpos específicos antipéptido en la infección por Trypanosoma cruzi. Dada la diversidad de proteínas presentes en los estadios del parásito, solo algunos antígenos se han estudiado en detalle. Además, se conoce poco acerca de la especificidad y funcionalidad de los anticuerpos que se generan durante la infección. En general, los anticuerpos cumplen diversas funciones: activan el sistema del complemento, mejoran la actividad de los fagocitos frente al parásito, inducen la citotoxicidad celular dependiente de anticuerpos e inhiben la infección de las células huéspedes (Lages-Silva, et al., 1987; Lima-Martins, et al., 1985; Wirth \& Kierszenbaum, 1987). Es así como en modelos múridos de infección aguda, se ha determinado que los 
anticuerpos contra las transialidasas (TS) parasitarias reducen la parasitemia y la mortalidad mediante el uso de anticuerpos policlonales (Krettli \& Brener, 1976), así como de un anticuerpo monoclonal anti-TS (Franchin, et al., 1997).

En nuestros estudios se ha empleado como antígeno una proteína de membrana del cinetoplasto con un peso molecular de $11 \mathrm{kDa}$, la KMP-11, la cual está muy conservada en parásitos como los tripanosomas y las leishmanias (Thomas, et al., 2000). Aunque la función de esta proteína no se conoce, su ausencia en el parásito impide la división, como en el caso de T. brucei (Li \& Wang, 2008). En T. cruzi, la proteína KMP-11 está asociada a proteínas del citoesqueleto, por lo cual se ha postulado que puede estar involucrada en la motilidad del parásito en su estado flagelar (Thomas, et al., 2000). La proteína KMP-11 se expresa en todas las formas del ciclo de vida del parásito y es reconocida en sueros de pacientes en fase aguda y crónica, aunque con algunas diferencias: en los pacientes con infección aguda presenta niveles de anticuerpos más bajos, con predominio de los isotipos IgG3 e IgG4, en tanto que en pacientes con infección crónica se presenta en niveles más elevados y es reconocida principalmente por los isotipos IgG1 e IgG3, los cuales presentan mayor unión a receptores FC para IgG (FC $\gamma \mathrm{R}$ ) (Flechas, et al., 2009), mejorando así las funciones efectoras de los fagocitos. En estudios de mapeo de epítopes $\mathrm{B}$ se ha demostrado que los anticuerpos de pacientes infectados reconocían más la región N-terminal de la proteína (Thomas, et al., 2001), sin embargo, es importante señalar que en ratones transgénicos que expresan la molécula HLA* A-0201 se detectó la presencia de un epítope restringido a esta molécula de clase I humana, reconocido por linfocitos T CD8+ (Marañón, et al., 2001). Este epítope de nueve aminoácidos (TLEEFSAKL), localizado en la región N-terminal, posición 4-12, se denominó inicialmente como K1, pero posteriormente se lo denominó como TcTLE (Finkelsztein, et al., 2015). Aunque los péptidos cortos toman una estructura al azar en soluciones, el TcTLE adquiere una estructura de hélice alfa, lo que permitiría su reconocimiento por parte de los anticuerpos (Figura 1). Este péptido es reconocido en mayor porcentaje (56\%) en los sueros de individuos sintomáticos con enfermedad de Chagas que en aquellos de personas asintomáticas (28 \%), con un predominio del isotipo IgG1 (Diez, et al., 2007). Dado que no se conoce la función de estos anticuerpos anti-péptido, el siguiente paso se centró en el estudio experimental in vitro de anticuerpos anti-TcTLE. En suero policlonal de conejos inmunizados con el péptido en adyuvante de Freund, se obtuvo un bajo título de anticuerpos, sin embargo, estos anticuerpos fueron capaces de reconocer la proteína recombinante KMP$11 \mathrm{y}$ la proteína nativa presente en las formas flageladas (epimastigotes y tripomastigotes) del parásito (Diaz-Soto, et al., 2012). En un segundo ensayo de inmunización, la producción de anticuerpos anti-TcTLE aumentó al inmunizar conjuntamente con un péptido inmunomodulador no derivado del parásito. Los anticuerpos policlonales generados reconocieron igualmente los amastigotes intracelulares. En los dos sueros policlonales obtenidos se demostró la habilidad de inhibir la infección de células in vitro, tanto en un cultivo de células Vero (Diaz-Soto, et al., 2012) como en un modelo de astrocitoma humano infectado con T. cruzi (VargasZambrano, et al., 2013). En este último, se observó que los anticuerpos alteraban la motilidad de los tripomastigotes afectando su capacidad de invasión e infección (Finkelsztein, et al., 2015).

En resumen, al péptido TcTLE se unen anticuerpos de sueros de pacientes con enfermedad de Chagas, lo cual implica que se trata de un epítope B reconocido de forma natural durante la infección. En modelos de infección in vitro se pudo demostrar que los anticuerpos anti-péptidos son capaces de reconocer la proteína nativa en las formas parasitarias e inhibir la entrada de tripomastigotes a los cultivos celulares. Parte de esta inhibición se atribuye a la alteración del movimiento flagelar de los tripomastigotes.

La inmunidad celular adquirida en respuesta a la infección crónica por Trypanosoma cruzi. Existen dos subpoblaciones principales de linfocitos T según sus marcadores y su funcionalidad, la de linfocitos T CD4+, o ayudadores, y la de CD8+, o citotóxicos, ambas implicadas en el control de la infección por T. cruzi. En un modelo de infección aguda en ratones, la eliminación de los linfocitos T CD8+ incrementó la parasitemia y la mortalidad comparado con los ratones control (Tarleton, 1980), en tanto que en los humanos, la disminución de los linfocitos CD4+, como ocurre en la infección por HIV, permite la reactivación del parásito (de Freitas, et al., 2011), lo cual indica que los linfocitos $\mathrm{T}$ son cruciales en el control de la infección. Los linfocitos $\mathrm{T}$ circulan por el sistema linfoide como linfocitos vírgenes cuyo objetivo es encontrar un antígeno en los

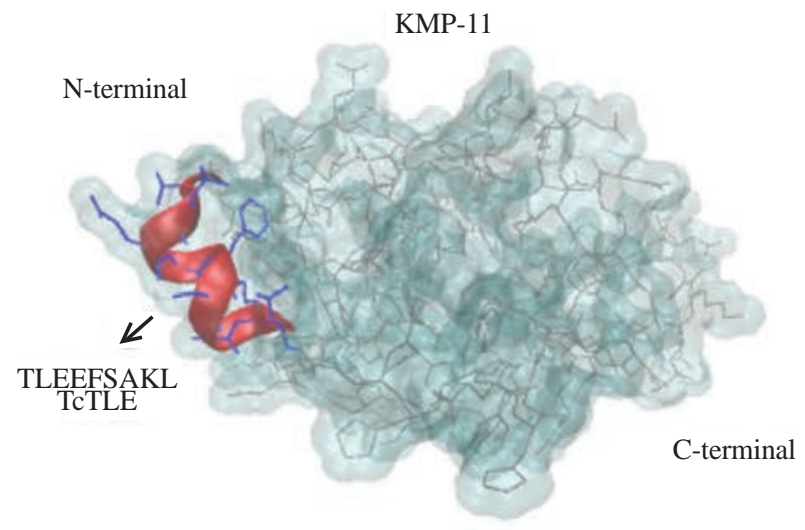

Figura 1. Modelo bioinformático del péptido TcTLE en la proteína KMP-11. Los aminoácidos 4 a 12 que representan el péptido se muestran como cinta (en rojo), con sus respectivas cadenas laterales (en azul). El resto de los aminoácidos en la proteína se muestran en gris. Imagen generada con el programa Visual Molecular Dynamics (VMD) http://www.ks.uiuc.edu/Research/vmd/ y elaborada con la colaboración del profesor Manu Forero-Shelton, Ph.D., Grupo de Biofísica, Universidad de los Andes, Bogotá 
órganos linfoides secundarios. Si los linfocitos vírgenes reconocen un antígeno a través de su receptor TCR y se diferencian a linfocitos $\mathrm{T}$ efectores. Existen diversas poblaciones de linfocitos efectores que se localizan en los tejidos donde ejercen sus funciones, como la producción de las citocinas INF- $\gamma$ o TNF- $\alpha$, entre otras, así como la producción de moléculas citotóxicas como la perforina. Una vez eliminado el agente infeccioso, como en el caso de algunas infecciones agudas, los linfocitos $\mathrm{T}$ efectores mueren por apoptosis y una pequeña población se convierte en linfocitos $\mathrm{T}$ de memoria. Al ser activados nuevamente por el antígeno, estos tienen la capacidad de generar nuevos linfocitos T efectores (Manhke, et al., 2013). Este patrón de diferenciación y función de los linfocitos T, en el caso de los T CD8+, se ve alterado en procesos como las infecciones crónicas. Tal es el caso de la infección por T. cruzi, ya que el parásito puede persistir en los individuos infectados. Debido a este fenómeno de alteración de la función de los linfocitos $\mathrm{T}$, conocido como agotamiento celular, la persistencia del antígeno induce cambios funcionales como la suspensión de la secreción de citocinas, y si bien los linfocitos $\mathrm{T}$ son específicos de antígeno, no pueden ejercer sus funciones efectoras adecuadamente. Por ejemplo, se disminuye la secreción de citocinas como la IL-12 y, por ende, la capacidad de proliferación, y, además, se producen cambios en la expresión de las moléculas, los receptores inhibitorios y los factores de transcripción (Wherry \& Kurachi, 2015). Se resumirá a continuación el trabajo en torno a la respuesta mediada por los linfocitos T, principalmente la de los CD8+ y, en menor proporción, los estudios con linfocitos T CD4+ y linfocitos T doblemente positivos (T CD4+/CD8+).

Respuesta de los linfocitos $T$ CD4+ en pacientes con enfermedad de Chagas crónica. Los linfocitos T CD4+ se caracterizan por tener diferentes tipos de subpoblaciones (Th) según el patrón de citocinas secretadas. Por ejemplo, los linfocitos Th1 secretan principalmente IFN- $\gamma$ y los linfocitos Th2 secretan IL-4, entre otras poblaciones, incluidas Th9, Th17, Th22, Tregs y ThF o foliculares. Las poblaciones de linfocitos Th se asocian a su función efectora, por ejemplo, los Th1 son importantes para el control de infecciones intracelulares, mientras que los Th2 lo son para el control de antígenos extracelulares y de helmintos (Schmitt \& Ueno, 2015). En los linfocitos CD4+ de sangreperiférica depacientes con cardiopatía chagásica crónica se encontró que después de la estimulación con una antígeno policlonal los linfocitos recientemente activados, o CD69+, producían una cantidad significativamente menor de IFN- $\gamma$ que los linfocitos $\mathrm{T}$ de individuos de control sin infección, aunque sin diferencias entre pacientes asintomáticos y sintomáticos. Igualmente, los linfocitos T CD4+ de estos pacientes (5 de 13 evaluados) produjeron IFN- $\gamma$ y no IL-4 en presencia de la proteína recombinante KMP-11 de $T$. cruzi y no hubo diferencias de acuerdo al estadio clínico de la enfermedad (Cuellar, et al., 2009). Los pacientes con enfermedad de Chagas crónica presentaron linfocitos T CD4+ con marcadores de activación crónica, como CD38 y HLA-DR, y disminuyeron la expresión del coestimulador CD28, molécula asociada a la activación posterior a la presentación antigénica. Estos cambios fueron más significativos en los pacientes asintomáticos que en los sintomáticos, y se asociaron con una menor expresión de la cadena zeta (z) del CD3. Dicha cadena contiene tres residuos de fosforilación importantes para la cascada de señalización intracelular que conlleva a la activación del linfocito $\mathrm{T}$ mediada por la producción de IL-2 (Giraldo, et al., 2013). La menor producción de citocinas ante el estímulo policlonal y la disminución de moléculas importantes en la señalización intracelular de los linfocitos, sugieren que la inmunidad celular se altera durante la infección crónica por T. cruzi. Asimismo, en otros estudios se ha demostrado que los linfocitos T CD4+ de pacientes con infección crónica producen menos INF- $\gamma$, lo cual se ha asociado con la gravedad de la cardiopatía y con predominio de células efectoras y expresión de marcadores de diferenciación temprana (CD27+CD28+) (Albareda, et al., 2009); en tanto que la respuesta polifuncional, medida como la secreción de INF- $\gamma$, TNF- $\alpha$ y la expresión de CD154, es mayor en niños infectados que en adultos con infección crónica, quienes presentan un respuesta monofuncional (Albareda, et al., 2013).

Linfocitos $T$ CD8+ específicos de antígeno de Trypanosoma cruzi y del péptido TcTLE. Como ya se mencionó, el péptido TLEEFSAKL o TcTLE de la región N-terminal de la proteína KMP-11 ha sido descrito como un epítope de unión a la molécula HLA-A*0201 (Marañón, et al., 2001). Este péptido fue reconocido por linfocitos $\mathrm{T}$ CD8+ provenientes de pacientes con enfermedad de Chagas crónica mediante un ensayo ELISpot para IFN- $\gamma$ en cerca de 17 \% de los individuos evaluados (Diez, et al., 2006). Una vez se demostró que el péptido TcTLE era procesado y presentado naturalmente en las moléculas del HLA durante el curso de la infección por T. cruzi, el siguiente paso fue el uso de tetrámeros fluorescentes HLA-A*0201 cargados con el péptido para determinar la frecuencia de células específicas durante la infección crónica mediante citometría de flujo. En total, 15 de los 19 pacientes crónicos evaluados presentaron linfocitos $\mathrm{T}$ CD8+ específicos del tetrámero en frecuencias que variaron entre 0,09 y 0,34 \%, sin diferencias por estadio de la enfermedad. Las células específicas presentaron un predominio del fenotipo de células efectoras $\left(\mathrm{T}_{\mathrm{EM}}\right)$ CD62L-/ CCR7- frente a las células de memoria $\left(\mathrm{T}_{\mathrm{CM}}\right), \mathrm{CD} 62 \mathrm{~L}+/$ CCR7+, y aunque en células de pacientes se detectaron la IL-2 y la IFN- $\gamma$, respectivamente, los porcentajes de expresión de estas citocinas entre los linfocitos T CD8+ específicos del tetrámero fueron muy bajos ( $<6 \%$ ) (Lasso, et al., 2010). Esta ausencia de secreción de citocinas por parte de las células específicas de antígeno puede ser el resultado de la disfunción celular en la enfermedad de Chagas y podría ser específica de la infección parasitaria o de otras respuestas celulares contra microorganismos dentro del mismo individuo. Por lo tanto, se evaluó la respuesta de los 
linfocitos T CD8+ frente a un epítope conocido del virus de la influenza igualmente restringido al HLA-A*0201. No se encontraron diferencias entre la frecuencia de las células del virus de influenza, el fenotipo y la secreción de citocinas entre pacientes infectados y no infectados por $T$. cruzi dentro del compartimento CD8+, lo que indica que la alteración se presenta en las células específicas de antígenos parasitarios (Lasso, et al., 2012). De igual forma, se observó que los linfocitos T CD8+ de pacientes con enfermedad de Chagas presentaron un aumento de la expresión de marcadores de activación, como CD38+ y HLA-DR, acompañado de una disminución de la expresión de membrana de CD28 y de la cadena CD3z intracelular. Sin embargo, a pesar de expresar marcadores de activación, estas células proliferan menos en presencia de un mitógeno como la fitohemaglutinina (PHA). Usando un modelo in vitro, se demostró que los linfocitos $\mathrm{T}$, tanto CD4+ como CD8+, provenientes de individuos no infectados disminuyeron drásticamente su proliferación en presencia de formas de tripomastigotes del parásito (Giraldo, et al., 2013). La poca respuesta de los linfocitos T en presencia del antígeno parasitario se ha asociado a una respuesta alterada a la IL-2 (Beltz \& Kierszenbaum, 1988). Igualmente, en linfocitos T CD8+, la producción de IFN- $\gamma$ en respuesta a los antígenos de $T$. cruzi se ve disminuida con la gravedad de la enfermedad (Laucella, et al., 2004).

Los linfocitos $T$ doblemente positivos en la enfermedad de Chagas crónica. Los linfocitos doblemente positivos, que expresan tanto CD4 como CD8, constituyen una subpoblación celular que se encuentra en sangre periférica en personas sanas en un porcentaje inferior a $3 \%$. Esta población no parece representar una población de linfocitos doblemente positivos escapados de la selección tímica, al parecer, se asocia a una activación de la transcripción de los genes correspondientes en sangre periférica. Estas células aumentan en diferentes enfermedades como las infecciones virales crónicas, los tumores e, inclusive, las alergias (Overgaard, et al., 2015). En el caso de los pacientes con enfermedad de Chagas crónica, esta subpoblación de linfocitos $\mathrm{T}$ está aumentada en sangre periférica comparada con la de individuos sanos y expresan marcadores de activación crónica como CD38 y HLA- DR. Los linfocitos $\mathrm{T}$ doblemente positivos en pacientes con enfermedad de Chagas crónica han presentado especificidad contra el péptido TcTLE, evaluada mediante tetrámeros fluorescentes HLA-A*0201 cargados con el péptido. Entre 3 y $5 \%$ de las células doblemente positivas se unió al tetrámero en individuos asintomáticos y sintomáticos, respectivamente. Cerca de $10 \%$ de ellas expresaron perforina intracelular, acompañada de marcadores de degranulación (CD107), así como un nivel muy bajo de secreción de IFN- $\gamma$ (Giraldo, et al., 2011). Estos resultados indican que los linfocitos doblemente positivos presentaron especificidad antigénica y capacidad citotóxica frente a los antígenos parasitarios.

En la Tabla 1 se presenta un resumen de los principales hallazgos en los estudios realizados en torno a la respuesta inmunitaria específica contra T. cruzi tanto de anticuerpos específicos como de linfocitos T. Los linfocitos T CD4+ y CD8+ en sangre periférica presentaron marcadores que indican una sobre-activación, acompañados de una disminución de la expresión de la cadena CD3z. Los linfocitos T con estas características, al igual que los linfocitos $\mathrm{T}$ doblemente positivos, se detectaron en el infiltrado de pacientes

Tabla 1. Resumen de los estudios en torno a la respuesta inmunitaria adaptativa en la infección por Trypanosoma cruzi

\begin{tabular}{|c|c|c|c|c|}
\hline & Modelo & Medición & Principal hallazgo & Comentario \\
\hline \multirow[t]{3}{*}{ Anticuerpos } & \multirow[t]{2}{*}{ Ex vivo } & Anti-KMP11 & $\begin{array}{l}\text { Reconocimiento por pacientes chagásicos } \\
\text { Isotipo IgG1 e IgG3 }\end{array}$ & Más en pacientes crónicos que en agudos \\
\hline & & Anti-TcTLE & $\begin{array}{l}\text { Reconocimiento por pacientes crónicos } \\
\text { Isotipo IgG1 }\end{array}$ & Mayor en sintomáticos \\
\hline & In vitro & Anti-TcTLE & $\begin{array}{l}\text { Anticuerpos anti-péptidos que reconocen las } \\
\text { formas parasitarias }\end{array}$ & Inhibición de motilidad e invasión \\
\hline \multirow[t]{5}{*}{ Linfocitos T } & \multirow[t]{4}{*}{ Ex vivo } & CD4+ & $\begin{array}{l}\text { Producción de IFN- } \gamma \text { en respuesta al estímulo } \\
\text { policlonal y ante antígeno parasitario }\end{array}$ & $\begin{array}{l}\text { Menor producción que en no infectados } \\
\text { Sin diferencias según el grado de enfermedad }\end{array}$ \\
\hline & & CD8+ & $\begin{array}{l}\text { Frecuencia de células específicos de TcTLE } \\
\text { menor de } 0,4 \%\end{array}$ & $\begin{array}{l}\text { Baja producción de citocinas como IFN- } \gamma \text { e } \\
\text { IL-2 }\end{array}$ \\
\hline & & $\begin{array}{l}\text { Doblemente } \\
\text { positivos }\end{array}$ & $\begin{array}{l}\text { Aumento en pacientes con enfermedad de } \\
\text { Chagas crónica } \\
\text { Detectados en corazón. }\end{array}$ & $\begin{array}{l}\text { Con especificidad antigénica y capacidad } \\
\text { citotóxica }\end{array}$ \\
\hline & & CD3+ & $\begin{array}{l}\text { Aumento de marcadores de activación } \\
\text { Disminución de moléculas coestimuladoras } \\
\text { Disminución de moléculas de señalización }\end{array}$ & $\begin{array}{l}\text { CD38 y HLA-DR. } \\
\text { CD28 } \\
\text { CD3z }{ }^{\mathrm{a}}\end{array}$ \\
\hline & In vitro & CD3+ & $\begin{array}{l}\text { Disminución de la proliferación en presencia } \\
\text { del parásito }\end{array}$ & Baja proliferación frente a mitógenos (PHA) \\
\hline
\end{tabular}

a. CD3z: cadena zeta intracelular; b. PHA: fitohemaglutinina 
con miocardiopatía chagásica, y no se observaron en tejidos cardiacos derivados del trasplante en pacientes con cardiopatía crónica no chagásica (Giraldo, et al., 2011 \& 2013). Dichos resultados sugieren que la alteración en la respuesta de los linfocitos T puede estar mediada por la persistencia del antígeno parasitario en los individuos infectados, lo cual altera la activación celular y contribuye a la patogenia en la enfermedad crónica (Figura 2).

\section{Infiltrado celular en la miocardiopatía chagásica crónica}

En los estudios histológicos de la cardiopatía chagásica crónica se encuentra miocitolisis, infiltrado celular, fibrosis intersticial y adelgazamiento de la membrana basal de los cardiomiocitos (Ferrans, et al., 1988). Mediante histoquímica en corazones de pacientes con cardiopatía chagásica crónica se han detectado antígenos parasitarios en $68 \%$ de los individuos (Belloti, et al., 1996), y mediante pruebas de biología molecular, en 85 \% (Jones, et al., 1993). El infiltrado celular se aprecia usualmente en parches con predominio de linfocitos T (Milei, et al., 1996), especialmente T CD8+ y CD4+ (Benvennuti, et al., 2000). Los linfocitos T CD8+ pueden participar en la patogenia a través de la citotoxicidad directa o mediante la producción de citocinas como TNF- $\alpha$ (RochaRodrigues, et al., 2012; Silverio, et al., 2012). En pacientes con mayor compromiso cardiaco se presentan altos niveles circulantes de TNF- $\alpha$ (Talvani, et al., 2004), los cuales se asocian con la progresión de la cardiopatía chagásica (Sousa, et al., 2014). Los linfocitos $T$ infiltrantes en miocardiopatía crónica expresan receptores inhibitorios (CTLA-4 y PD-1) indicadores de agotamiento celular (Argüello, et al., 2012). Vale la pena señalar que algunos de los cambios descritos son similares a los encontrados en linfocitos $\mathrm{T}$ de sangre periférica en pacientes con cardiopatía chagásica avanzada, los cuales también presentan características de agotamiento clonal, como la pérdida de la multifuncionalidad y la expresión de receptores inhibitorios (Giraldo, et al., 2013; Lasso, et al., 2015; Mateus, et al., 2017). Algunas de estas alteraciones fenotípicas y funcionales en los linfocitos $\mathrm{T}$ pueden revertirse con el tratamiento anti-parasitario (Mateus, et al., 2017).

El estudio de la respuesta inmunitaria contra T. cruzi sigue siendo un reto de la investigación actual, en parte por la compleja historia natural de la infección y las diferencias de la respuesta inmunitaria adaptativa en la fase aguda y la fase crónica de la infección, además de las variaciones genéticas del parásito. Sin embargo, el estudio de la respuesta ante antígenos específicos ha permitido dilucidar algunas de las funciones de los anticuerpos y de los linfocitos T durante la infección, lo cual ha facilitado, además de la patogénesis durante la enfermedad, la selección de posibles antígenos candidatos a vacunas.

\section{Agradecimientos}

A todos los coautores de los artículos citados en este manuscrito, a los pacientes y los individuos de control sanos que participaron en los estudios, así como a las agencias finan-

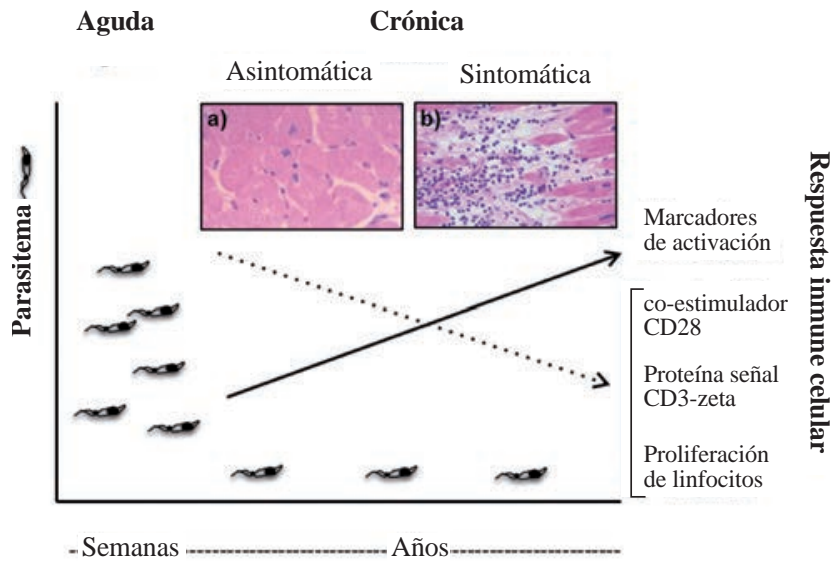

Figura 2. Esquema de los principales hallazgos en la respuesta inmunitaria celular. El eje de la $Y$ muestra la evolución de la enfermedad en el tiempo. En los ejes $X$ se muestran la parasitemia y el aumento (línea continua) o disminución (línea punteada) de los marcadores celulares evaluados. Las imágenes muestran biopsias cardiacas teñidas con hematoxilina y eosina de tejido sin infiltrado celular (a) y tejido con infiltrado de un paciente con enfermedad de Chagas crónica (b). Imágenes con 40x obtenidas con la colaboración de Ana María Uribe, MD-Patóloga, Departamento de Patología, Hospital Universitario San Ignacio, Bogotá

ciadoras, principalmente Colciencias, y las Vicerrectorías de Investigaciones de la Universidad de los Andes y de la Pontificia Universidad Javeriana.

\section{Contribución de los autores}

Todos los autores participaron en la concepción, la escritura, la revisión y la corrección del manuscrito.

\section{Declaración de conflicto de intereses}

Los autores no declaran ningún conflicto de intereses.

\section{Referencias}

Acevedo, G.R., Longhi, S.A., Bunying, A., Sabri, N., Atienza, A., Zago, M.P., Santos, R., Judkowski, V.A., Pinilla, C., Gómez, K.A. (2017). Methodological approach to the ex vivo expansion and detection of T. cruzi-specific T cells from chronic Chagas disease patients. PLoS One. 12: e0178380. doi: 10.1371/journal.pone.0178380.

Albareda, M.C., Olivera, G.C., Laucella, S.A., Álvarez, M.G., Fernández, E.R., Lococo, B., Viotti, R., Tarleton, R.L., Postan M. (2009) Chronic human infection with Trypanosoma cruzi drives CD4+ $\mathrm{T}$ cells to immune senescence. J Immunol. 183 (6): 4103-4108.

Albareda, M.C., De, Rissio, A.M., Tomas, G., Serjan, A., Álvarez, M.G., Viotti, R., Fichera, L.E., Esteva, M.I., Potente, D, Armenti, A., Tarleton, R.L., Laucella, S.A. (2013). Polyfunctional T cell responses in children in early stages of chronic Trypanosoma cruzi infection contrast with monofunctional responses of long-term infected adults. PLoS Negl Trop Dis. 7 (12): e2575

Argüello, R., Albareda, M., Álvarez, M., Bertocchi, G., Armenti, A., Vigliano, C., Meckert, P., Tarleton, R., Laucella, S. (2012). Inhibitory Receptors Are Expressed by 
Trypanosoma cruzi-Specific Effector T Cells and in Hearts of Subjects with Chronic Chagas Disease. PLoS ONE. 7: p.e35966

Barrias, E.S., de Carvalho, T.M., De Souza, W. (2013). Trypanosoma cruzi: Entry into Mammalian Host Cells and Parasitophorous Vacuole Formation. Front Immunol. 4: 186. doi: 10.3389/fimmu.2013.00186. eCollection 2013.

Bellotti, G., Bocchi, E.A., de Moraes, A.V., Higuch,i M.L,, Barbero-Marcial, M., Sosa, E., Esteves-Filho, A., Kalil, R., Weiss, R., Jatene, A., Pileggi, F. (1996) In vivo detection of Trypanosoma cruzi antigens in hearts of patients with chronic Chagas' heart disease. Am Heart J. 131: 301-307.

Benvenuti, L.A., Higuchi, M.L., Reis, M.M. (2000). Upregulation of adhesion molecules and class I HLA in the myocardium of chronic chagasic cardiomyopathy and heart allograft rejection, but not in dilated cardiomyopathy. Cardiovasc Pathol. 9: 111-117.

Bern, C., Montgomery, S.P., Herwaldt, B.L., Rassi, A. Jr., MarinNeto, J.A., Dantas, R.O., Maguire, J.H., Acquatella, H., Morillo, C., Kirchhoff, L.V., Gilman, R.H., Reyes, P.A., Salvatella, R., Moore, A.C. (2007). Evaluation and treatment of Chagas disease in the United States: A systematic review. JAMA. 298: 2171-2181.

Bern C. (2015). Chagas' Disease. N Engl J Med. 373: 456-466. doi: 10.1056/NEJMra1410150.

Beltz, L.A., Kierszenbaumm F. (1987). Suppression of human lymphocyte responses by Trypanosoma cruzi. Immunology. 60 (2): 309-315.

Brener, Z. (1973). Biology of Trypanosoma cruzi. Annu Rev Microbiol. 27: 347-382.

Campo, V.L., Martins-Teixeira, M.B., Carvalho, I. (2016). Trypanosoma cruzi Invasion into Host Cells: A Complex Molecular Targets Interplay. Mini Rev Med Chem. 16: 1084-1097.

Castillo-Riquelme, M., Guhl, F., Turriago, B., Pinto, N., Rosas, F., Martínez, M.F., Fox-Rushby, J., Davies, C., CampbellLendrum, D. (2008). The costs of preventing and treating Chagas disease in Colombia. PLoS Negl Trop Dis. 2: e336. doi: 10.1371/journal.pntd.0000336.

Cuéllar, A., Santander, S.P., Thomas, M.C., Guzmán, F., Gómez, A., López, M.C., Puerta, C.J. (2008). Monocytederived dendritic cells from chagasic patients vs healthy donors secrete differential levels of IL-10 and IL- 12 when stimulated with a protein fragment of Trypanosoma cruzi heat-shock protein-70. Immunol Cell Biol. 86: 255-260.

Cuéllar, A., Rojas, F., Bolaños, N., Díez, H., Thomas, M.C., Rosas, F., Velasco, V., López, M.C., González, J.M., Puerta, C. (2009). Natural CD4(+) T-cell responses against Trypanosoma cruzi KMP-11 protein in chronic chagasic patients. Immunol Cell Biol. 87: 149-153. doi: 10.1038/ icb.2008.76. Erratum in: Immunol Cell Biol. 2009. 7: 108.

de Freitas, V.L., da Silva, S.C., Sartori, A.M., Bezerra, R.C., Westphalen, E.V., Molina, T.D., Teixeira, A.R., Ibrahim, K.Y., Shikanai-Yasuda, M.A. (2011). Real-time PCR in HIV/Trypanosoma cruzi coinfection with and without Chagas disease reactivation: Association with HIV viral load and CD4 level. PLoS Negl Trop Dis. 5: e1277. doi: 10.1371/journal.pntd.0001277

de Souza, W., de Carvalho, T.M., Barrias, E.S. (2010) Review on Trypanosoma cruzi: Host Cell Interaction. Int J Cell Biol. 2010. pii: 295394. doi: 10.1155/2010/295394
Dey, N., Sinha, M., Gupta, S., González, M.N., Fang, R., Endsley, J.J., Luxon, B.A., Garg, N.J. (2014). Caspase-1/ASC inflammasome-mediated activation of IL-1 $\beta$-ROS-NF- $\mathrm{BB}$ pathway for control of Trypanosoma cruzi replication and survival is dispensable in NLRP3-/- macrophages. PLoS One. 9: e 111539.

Díaz-Soto, J, C., Lasso, P., Guzmán, F., Forero-Shelton, M., Thomas, M.C., López, M.C., Guhl, F., Cuéllar, A., Puerta, C.J., González, J.M. (2012). Rabbit serum against K1 peptide, an immunogenic epitope of the Trypanosoma cruzi KMP-11, decreases parasite invasion to cells. Acta Trop. 123: 224-229. doi: 10.1016/j.actatropica.2012.05.015.

Díez, H., López, M.C., Thomas, M.C., Guzmán, F., Rosas, F., Velazco, V., González, J.M., Puerta, C. (2006). Evaluation of IFN-gamma production by CD8 T lymphocytes in response to the K1 peptide from KMP-11 protein in patients infected with Trypanosoma cruzi. Parasite Immunol. 28: 101-105.

Díez, H., Guzmán, F., Alba, M.P., Cuéllar,A., Thomas, M.C., López, M.C., Rosas, F., Velasco, V., González, J.M., Patarroyo, M.E., Puerta, C.J. (2007). Immunological and structural characterization of an epitope from the Trypanosoma cruzi KMP-11 protein. Peptides. 28: 1520-1526.

Dos-Santos, A.L., Carvalho-Kelly, L.F., Dick, C.F., MeyerFernandes, J.R. (2016). Innate immunomodulation to trypanosomatid parasite infections. Exp Parasitol. 167: 6775. doi: 10.1016/j.exppara.2016.05.005.

Ferrans, V.J., Milei, J., Tomiata, Y., Storino, R.A. (1988). Basement membrane thickening in cardiac myocytes and capillaries in chronic Chagas' disease. Am J Cardiol. 61: 1137-1140.

Finkelsztein, E.J., Díaz-Soto, J.C., Vargas-Zambrano, J.C., Suesca, E., Guzmán, F., López, M.C., Thomas, M.C., Forero-Shelton, M., Cuéllar, A., Puerta, C.J., González, J.M. (2015). Altering the motility of Trypanosoma cruzi with rabbit polyclonal anti-peptide antibodies reduces infection to susceptible mammalian cells. Exp Parasitol. 150: 3643. doi: 10.1016/j.exppara.2015.01.007

Flechas, I.D., Cuéllar, A., Cucunubá, Z.M., Rosas, F., Velasco, V., Steindel, M., Thomas, M.C., López, M.C., González, J.M., Puerta C.J. (2009). Characterising the KMP-11 and HSP-70 recombinant antigens' humoral immune response profile in chagasic patients. BMC Infect Dis. 25: 186. doi: 10.1186/1471-2334

Franchin, G., Pereira-Chioccola, V.L., Schenkman, S., Rodrigues, M.M. (1997). Passive transfer of a monoclonal antibody specific for a sialic acid-dependent epitope on the surface of Trypanosoma cruzi trypomastigotes reduces infection in mice. Infect. Immun. 65: 2548-2554.

Giraldo, N.A., Bolaños, N.I., Cuéllar, A., Guzmán, F., Uribe, A.M., Bedoya, A., Olaya, N., Cucunubá, Z.M., Roa, N., Rosas, F., Velasco, V., Puerta, C.J., González, J.M. (2011). Increased CD4+/CD8+ double-positive T cells in chronic Chagasic patients. PLoS Negl Trop Dis. 5: e1294. doi: 10.1371/journal.pntd.0001294.

Giraldo, N.A., Bolaños, N.I., Cuéllar, A., Roa, N., Cucunubá, Z., Rosas, F., Velasco, V., Puerta, C.J., González, J.M. (2013). T lymphocytes from chagasic patients are activated but lack proliferative capacity and down-regulate CD28

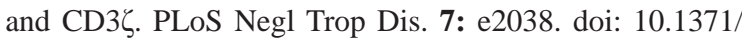
journal.pntd.0002038 
Klahr, J.I., Uribe, A.M., Roa, N., González, J.M. (2016). Inmunidad celular en la patogénesis de la cardiopatía chagásica crónica. Rev Col Cardiol. 23: 568-575. doi: 10.1016/j.rccar. 2016.04.017

Krettli, A.U., Brener, Z. (1976). Protective effects of specific antibodies in Trypanosoma cruzi infections. J. Immunol. 116: $755-760$.

Lages-Silva, E., Ramírez, L.E., Krettli, A.U., Brener, Z. (1987). Effect of protective and non-protective antibodies in the phagocytosis rate of Trypanosoma cruzi blood forms by mouse peritoneal macrophages. Parasite Immunol. 9: 21-30.

Lasso, P., Mesa, D., Cuéllar, A., Guzmán, F., Bolaños, N., Rosas, F., Velasco, V., Thomas, M.C., López, M.C., González, J.M., Puerta, C.J. (2010). Frequency of specific CD8+ T cells for a promiscuous epitope derived from Trypanosoma cruzi KMP-11 protein in chagasic patients. Parasite Immunol. 32: 494-502. doi: 10.1111/j.1365-3024. 2010.01206.x

Lasso, P., Mesa, D., Bolaños, N., Cuéllar, A., Guzmán, F., Cucunubá, Z., Rosas, F., Velasco, V., Thomas, M.C., López, M.C., González, J.M., Puerta, C.J. (2012). Chagasic patients are able to respond against a viral antigen from influenza virus. BMC Infect Dis. 12: 198. doi: 10.1186/1471-2334-12-198

Lasso, P., Mateus, J., Pavía, P., Rosas, F., Roa, N., Thomas, M.C., López, M.C., González, J.M., Puerta, C.J., Cuéllar, A. (2015). Inhibitory Receptor Expression on CD8+ T Cells Is Linked to Functional Responses against Trypanosoma cruzi Antigens in Chronic Chagasic Patients. J Immunol. 195 (8): 3748-3758. doi: 10.4049/jimmunol.1500459

Laucella, S.A., Postan, M., Martin, D., Hubby Fralish, Bm, Albareda, M, C., Álvarez, M.G., Lococo, B., Barbieri, G., Viotti, R.J., Tarleton, RL. (2004). Frequency of interferongamma -producing T cells specific for Trypanosoma cruzi inversely correlates with disease severity in chronic human Chagas disease. $J$ Infect Dis. 189: 909-918.

Ley, V., Robbins, E.S., Nussenzweig, V., Andrews, N.W. (1990). The exit of Trypanosoma cruzi from the phagosome is inhibited by raising the $\mathrm{pH}$ of acidic compartments. J Exp Med. 171: 401-413.

Li, Z., Wang, C. (2008). KMP-11, a Basal Body and Flagellar Protein, Is Required for Cell Division in Trypanosoma brucei. Eukaryotic Cell. 7: 1941-1950.

Lima-Martins, M.V., Sánchez, G.A., Krettli, A.U., Brener, Z. (1985). Antibody-dependent cell cytotoxicity against Trypanosoma cruzi is only mediated by protective antibodies. Parasite Immunol. 7: 367-376.

Magalhães, L.M., Viana, A., Chiari, E., Galvão, L.M., Gollob, K.J., Dutra W.O. (2015). Differential Activation of Human Monocytes and Lymphocytes by Distinct Strains of Trypanosoma cruzi. PLoS Negl Trop Dis. 9: e0003816. doi: 10.1371/journal.pntd.0003816

Magdesian, M.H., Giordano, R., Ulrich, H., Juliano, M.A., Juliano, L., Schumacher, R.I., Colli, W., Alves M.J. (2001). Infection by Trypanosoma cruzi. Identification of a parasite ligand and its host cell receptor. J Biol Chem. 276: 19382-19389.

Mahnke, Y.D., Brodie, T.M., Sallusto, F., Roederer, M., Lugli, E. (2013). The who's who of T-cell differentiation: Human memory T-cell subsets. Eur J Immunol. 43: 2797-2809. doi: 10.1002/eji.201343751
Marañón, C., Thomas, M.C., Planelles, L., López, M.C. (2001). The immunization of $\mathrm{A} 2 / \mathrm{K}(\mathrm{b})$ transgenic mice with the KMP11-HSP70 fusion protein induces CTL response against human cells expressing the T. cruzi KMP11 antigen: Identification of A2-restricted epitopes. Mol Immunol. 38: 279-287.

Mateus, J-, Pérez-Antón, E., Lasso, P., Egui, A., Roa, N., Carrilero, B., González, J.M., Thomas, M.C., Puerta, C.J., López, M.C., Cuéllar, A. (2017). Antiparasitic Treatment Induces an Improved CD8+ T Cell Response in Chronic Chagasic Patients. J Immunol. 198: 3170-3180. doi: 10.4049/jimmunol.1602095

Medeiros, N.I., Fares, R.C., Franco, E.P., Sousa, G.R., Mattos, R.T., Cháves, A.T., Nunes, M.D., Dutra, W.O., Correa-Oliveira, R., Rocha, M.O., Gomes, J.A. (2017). Differential Expression of Matrix Metalloproteinases 2, 9 and Cytokines by Neutrophils and Monocytes in the Clinical Forms of Chagas Disease. PLoS Negl Trop Dis. 11: e0005284. doi: 10.1371/journal.pntd.0005284

Milei, J., Fernández, A., Vanzulli, G., Storino, S., Matturri, R., Rossi, L. (1996). Myocardial inflammatory infiltrate in human chronic chagasic cardiomyopathy: Immunohistochemical findings. Cardiovasc Pathol. 5: 209-219.

Molina, H.A., Kierszenbaum, F. (1987). A study of human myocardial tissue in Chagas' disease: Distribution and frequency of inflammatory cell types. Int J Parasitol. 17: 1297-1305

Morillo, C.A., Marin-Neto, J.A., Avezum, A., Sosa-Estani, S., Rassi, A. Jr., Rosas, F., Villena, E., Quiroz, R., Bonilla, R., Britto, C., Guhl, F., Velázquez, E., Bonilla, L., Meeks, B., Rao-Melacini, P., Pogue, J., Mattos, A., Lazdins, J., Rassi, A., Connolly, S.J., Yusuf, S.; BENEFIT Investigators. (2015). Randomized Trial of Benzinidazole for Chronic Chagas' Cardiomyopathy. N Engl J Med. 373: 1295-306. doi: 10.1056/NEJMoa1507574

Noya, B.A., Díaz-Bello, Z., Colmenares, C., Ruiz-Guevara, R., Mauriello, L., Muñoz-Calderón, A., Noya, O. (2015). Update on oral Chagas disease outbreaks in Venezuela: Epidemiological, clinical and diagnostic approaches. Mem Inst Oswaldo Cruz. 110: 377-386. doi: 10.1590/007402760140285

Overgaard, N.H., Jung, J.W., Steptoe, R.J., Wells, J.W. (2015). CD4+/CD8+ double-positive T cells: More than just a developmental stage? J Leukoc Biol. 97: 31-38. doi: 10.1189/jlb.1RU0814-382.

Rassi, A., Rezende, J.M., Luquetti, A.O., Rassi, Jr. A. (2010). Clinical Phases and Forms of Chagas Disease. American Trypanosomiasis Chagas Disease. In: One Hundred Years of Research. p. 709-741. Disponible en: chttps://doi.org/10. 1016/B978-0-12-384876-5.00027-7

Rassi, A. Jr., Marin-Neto, J.A., Rassi. A. (2017). Chronic Chagas cardiomyopathy: A review of the main pathogenic mechanisms and the efficacy of aetiological treatment following the BENznidazole Evaluation for Interrupting Trypanosomiasis (BENEFIT) trial. Mem Inst Oswaldo Cruz. 112: 224-235. doi: 10.1590/0074-02760160334

Rocha-Rodrigues, D.B., dos Reis, M.A., Romano, A., Pereira, S.A., Teixeira, V.P., Tostes S. J.r, Rodrigues, V. Jr. (2012). In situ expression of regulatory cytokines by heart inflammatory cells in Chagas' disease patients with heart failure. Clin Dev Immunol. 2012: 361730. doi: 10.1155/2012/361730 
Rosas, F., Roa, N., Cucunubá, Z., Cuéllar, A., González, J.M., Puerta, C. (2012). Chagasic cardiomyopathy. In: Veselka J. Cardiomyopathies - From Basic Research to Clinical Management. Rijeka, Croatia: Editorial INTECH. p. 775-800.

Rosestolato, C.T., Dutra, J, M., De Souza, W., de Carvalho, T.M. (2002) Participation of host cell actin filaments during interaction of trypomastigote forms of Trypanosoma cruzi with host cells. Cell Struct Funct. 27: 91-98.

Sabino, E.C., Ribeiro, A.L., Salemi, V.M., Di Lorenzo Oliveira, C., Antunes, A.P., Menezes, M.M., Lanni, B.M., Nastari, L., Fernandes, F., Patavino, G.M., Sachdev, V., Capuani, L., de Almeida-Neto, C., Carrick, D.M., Wright. D., Kavounis, K., Gonçalez, T.T., Carneiro-Proietti, A.B., Custer, B., Busch, M.P., Murphy, E.L. (2013). Ten-year incidence of Chagas cardiomyopathy among asymptomatic Trypanosoma cruzi-seropositive former blood donors. Circulation. 127: 1105-1115. doi: 10.1161/ CIRCULATIONAHA.112.123612

Schmitt, N., Ueno. H. (2015). Regulation of human helper T cell subset differentiation by cytokines. Curr Opin Immunol. 34: 130-136. doi: 10.1016/j.coi.2015.03.007

Silverio, J.C., Pereira, I.R., Cipitelli, M.C., Vinagre, N.F., Rodrigues, M.M., Gazzinelli, R.T., Lannes-Vieira, J. (2012). CD8+ T-cells expressing interferon gamma or perforin play antagonistic roles in heart injury in experimental Trypanosoma cruzi-elicited cardiomyopathy. PLoS Pathog. 8: e1002645. doi: 10.1371/journal.ppat.1002645

Sousa, G.R., Gomes, J.A., Fares, R.C., Damásio, M.P., Chaves, A.T., Ferreira, K.S., Nunes, M.C., Medeiros, N.I., Valente, V.A., Corrêa-Oliveira, R., Rocha, M.O. (2014). Plasma cytokine expression is associated with cardiac morbidity in Chagas disease. PLoS One. 9: e87082. doi: 10.1371/journal.pone.0087082

Sosa-Estani, S., Segura, E.L., Ruiz, A.M., Velázquez, E., Porcel, B.M., Yampotis, C. (1998). Efficacy of chemotherapy with benzinidazole in children in the indeterminate phase of Chagas' disease. Am J Trop Med Hyg. 59: 526-529.

Talvani, A., Rocha, M.O., Barcelos, L.S., Gomes, Y.M., Ribeiro, A.L., Teixeira, M.M. (2004). Elevated concentrations of CCL2 and tumor necrosis factor-alpha in chagasic cardiomyopathy. Clin Infect Dis. 38: 943-950.
Tarleton, R. (1980). Depletion of CD8+ T cells increases susceptibility and reverses vaccine-induced immunity in mice infected with Trypanosoma cruzi. J Immunol. 144: 717-724.

Tarleton RL. (2015). CD8+ T cells in Trypanosoma cruzi infection. Semin Immunopathol. 37: 233-238. doi: 10.1007/s00281015-0481-9.

Thomas, M.C., García-Pérez, J.L., Alonso, C., López, M.C. (2000). Molecular characterization of KMP11 from Trypanosoma cruzi: A cytoskeleton-associated protein regulated at the translational level. DNA Cell Biol. 19: 47-57.

Thomas, M.C., Longobardo, M.V., Carmelo, E., Marañón, C., Planelles, L., Patarroyo, M.E., Alonso, C., López, M.C. (2001). Mapping of the antigenic determinants of the $T$. cruzi kinetoplastid membrane protein-11. Identification of a linear epitope specifically recognized by human Chagasic sera. Clin Exp Immunol. 123: 465-471.

Vargas-Zambrano, J.C., Lasso, P., Cuéllar, A., Puerta, C.J., González, J.M. (2013). A human astrocytoma cell line is highly susceptible to infection with Trypanosoma cruzi. Mem Inst Oswaldo Cruz. 108: 212-219.

Vitelli-Avelar, D.M., Sathler-Avelar, R., Massara, R.L., Borges, J.D., Lage, P.S., Lana, M., Teixeira-Carvalho, A., Dias, J.C., Elói-Santos, S.M., Martins-Filho, O.A. (2006). Are increased frequency of macrophage-like and natural killer (NK) cells, together with high levels of NKT and CD4+CD25high $\mathrm{T}$ cells balancing activated CD8+ T cells, the key to control Chagas' disease morbidity. Clin Exp Immunol. 145: 81-92.

Wherry, E.J., Kurachi, M. (2015). Molecular and cellular insights into T cell exhaustion. Nat Rev Immunol. 15: 486-499. doi: 10.1038/nri3862

Wirth, J.J., Kierszenbaum, F. (1987). Inhibition of mammalian host cell infection by insect-derived, metacyclic forms of Trypanosoma cruzi in the presence of human or rabbit anti-T. cruzi antibodies. Int. J. Parasitol. 17: 841-845. 